\title{
Access to Quality Diagnosis and Rational Treatment for TB: Evidence from China - Gates Tuberculosis Control Project Phase III
}

\section{Zhipeng Li}

Fudan University

\section{Wenhui Mao}

Duke Global Health Institute

\section{Fei Huang}

Chinese Center for Disease Control and Prevention

\section{Ni Wang}

Chinese Center for Disease Control and Prevention

\section{Liping Ma}

Beijing Chest Hospital

\section{Liqun Zhang}

Beijing Chest Hospital

\section{Mengqiu Gao}

Beijing Chest Hospital

\section{Weibing Wang}

Fudan University School of Public Health

Qi Zhao ( $\nabla$ zhaoqi@shmu.edu.cn)

School of Public Health, Fudan University https://orcid.org/0000-0003-0140-3920

\section{Shenglan Tang}

Duke Global Health Institute

\section{Research Article}

Keywords: Tuberculosis (TB), evaluation, TB diagnosis, treatment, rapid molecular test (RMT), second line drug

Posted Date: February 4th, 2021

DOl: https://doi.org/10.21203/rs.3.rs-169923/v1

License: (c) (1) This work is licensed under a Creative Commons Attribution 4.0 International License.

Read Full License 


\section{Abstract}

Introduction: Over the last ten years, China has shifted the tasks of TB clinical care from the national TB control program to TB designated hospitals. Although China has successfully maintained a moderate reduction in TB incidence during this transition, China continues to face many challenges in improving the quality of TB diagnosis and treatment provided by the hospitals. The Chinese National Health Commission (NHC) and Chinese Center for Disease Control and Prevention (China CDC) collaborated with the Bill and Melinda Gates Foundation (Gates Foundation) to implement a China NHC- Gates Foundation Tuberculosis Control Project that aimed to scale up a comprehensive TB control model to improve the quality of TB care in three provinces (Zhejiang, Jilin and Ningxia). In this paper, we evaluate whether the China NHC-Gates Foundation TB project has helped improve access to quality diagnosis and rational treatment of TB.

Methods: We selected 12 project counties as evaluation sites based on their economic development and TB notification status at the beginning of the project period in 2016. We conducted baseline and final evaluations in 2016 and 2019 respectively for data collection. We obtained TB patients' information from the TB Information Management System (TBIMS) and reviewed medical records of TB cases to assess access to quality diagnosis and rational treatment at TB designated hospitals. We examined the change in coverage of different diagnostic tests for diagnosing TB and drug susceptibility, including sputum smear tests, culture, traditional DST and rapid molecular tests (RMT). We also evaluated the proportion of TB patients receiving adequate diagnostic services according to China's National TB guidelines. For access to rational treatment, we evaluated the proportion of TB patients using unnecessary second-line drugs (SLDs) and the proportion of TB patients receiving adequate follow-up examinations during the entire treatment course. All indicators were analyzed by province.

Results: Overall, we observed improved access to diagnosis on multiple indicators. The percentage of sputum smear-negative patients taking culture or rapid molecular tests (RMT) doubled between 2015 and 2018 (from 35\% to 87\%). The percentage of bacteriologically confirmed pulmonary TB cases increased from $36 \%$ in 2015 to $52 \%$ in 2018 . RMT has been widely used and contributed an additional $20.3 \%$ of bacteriologically confirmed TB cases in 2018. The percentage of TB patients taking DST also doubled from 2015 to 2018 (from $40 \%$ to 82\%) while the proportion of TB patients receiving adequate diagnosis services increased from $88 \%$ in 2015 to $96 \%$ in 2018 . Among all smear-negative TB patients, over $86 \%$ received the recommended diagnostic services at the end of the study period, an improvement from $75 \%$ prior to project implementation. Asymptomatic smear-negative TB patients' diagnosis quality improved even more: $63.1 \%$ received the recommended services in 2018 , a massive improvement from only $18.2 \%$ prior to the project period. Although the proportion of patients receiving recommended follow-up tests during the entire treatment course improved, the proportion of TB patients treated by unnecessary SLDs increased from $12.6 \%$ in 2015 to $19.9 \%$ in 2018 . Despite progress, regional disparities remain among the provinces, albeit the gaps between them narrowed down for almost all indicators. 
Conclusions: Our study found that between 2015 to 2018, access to quality TB diagnosis and follow-up services has been improved substantially in the study sites, primarily due to the implementation of the China NHC- Gates Foundation Tuberculosis Control Project. Despite progress, irrational use of SLDs remains a problem because of perverse financial incentives given to the hospitals.

\section{Introduction}

Tuberculosis (TB) remains a global health concern and challenge. In 2017, TB caused the highest number of deaths and the second highest Disability-Adjusted Life Years (DALYs) among all communicable diseases globally. Combating TB has been prioritized by the Sustainable Development Goals by 2030 . The WHO End TB Strategy endeavors to reduce TB deaths by $95 \%$ and eliminate catastrophic health expenditures caused by TB by 2035[1,2]. China has one of the highest burdens of TB and remarkable achievements have been made to improve the control and care for TB[3] between 2000 and 2010. The Directly Observe Treatment Strategy (DOTS) has been scaled up across the country by the Chinese Centers for Disease Control and Prevention, and significant government subsidies have been provided to enable free first-line anti-TB medicines, smear tests and chest X-rays[4, 5]. These efforts have led to significant declines in China's incidence of TB[3].

TB clinical care has been integrated and shifted from the CDCs to TB designated hospitals since 2010. Despite this progress, the diagnosis and rational treatment of TB remain key issues of concern. In 2018 , only $37 \%$ of the TB notified cases in China were confirmed through bacteriological detection, much lower than the global average of 55\%[6]. Bacteriological detection of TB is essential for timely and precise diagnosis of TB and early initiation of treatment $[7,8]$. Yet the majority of TB patients in China are diagnosed clinically based on symptoms, chest radiography, suggestive histology and immunologic tests that are generally associated with low specificity for TB diagnosis. This could potentially delay treatment for TB or in contrast, lead to unnecessary treatment for those misdiagnosed. Another factor is the poor access to the universal drug susceptibility test (DST), which would help diagnose drug-resistant TB and provide timely treatment.

China has also seen growth in the prevalence of multidrug-resistant tuberculosis(MDR-TB) over time. In 2018, it had an estimated 66000 MDR-TB/RR-TB incidents, among which only $22.8 \%$ were laboratoryconfirmed, presenting a much lower MDR-TB detection rate compared to the global average of $39 \%$ in same year[6]. Low detection rate could simply lead to low treatment rate, and further spread of MDRTB/RR-TB[9].

In terms of treatment, a recent study found both over and under-treatment of TB in China[10]. Despite the free provision of first-line anti-TB medicines, poor compliance with TB treatment has been frequently reported while irrational use of medicines, especially second line anti-TB drug and liver protection drugs, have been increasingly observed[11-13]. In addition, follow-up examinations, which are outlined in China's guidelines, were not implemented according to the guidance. Follow up exams should include things like routine blood tests, liver and renal function tests to identity adverse drug reactions (ADR) in a 
timely manner to make proper adjustments in treatment. Yet these follow-up examinations were not implemented well, per the guideline.

Several studies have evaluated the significance of rapid molecular test (RMT) and proved that it has substantially improved the quality of TB diagnosis, with better sensitivity and specificity than traditional tests. Additionally, the testing time of RMT is shorter, resulting in more timely initiation of treatment for TB patients $[14,15]$. Despite this evidence, RMT has yet to replace other testing methods in China.

To improve TB control and care in China, the China National Health Commission and CDC established a partnership with the Bill \& Melinda Gates Foundation. From 2009 to 2012, RMT, along with standardized treatment based on resistance testing, was piloted in four prefectures of four provinces in China through the partnership with the Bill \& Melinda Gates Foundation (BMGF), also known as the first phase of the "China NHC-Gates Foundation TB project"[16, 17]. The second phase of the project was implemented from 2012 to 2015, with a focus on developing comprehensive TB control models for effective diagnosis, treatment and management of TB patients using RMT and delivery approaches. The third phase, The China National Health Commission-The Bill \& Melinda Gates Foundation TB Phase III program (China Gates Project Phase III), was initiated in 2016 for a three-year project period to implement a comprehensive TB control model including RMT, capacity training on TB diagnosis and clinic treatment, financing for TB treatment, information system and electronic medicine management system (EMMs). The model was scaled up in 172 countries in three provinces: Zhejiang, Jilin, Ningxia.

The purpose of this study was to evaluate the China NHC-Gates Foundation TB project (Phase III) on its impact on access to quality diagnosis and rational treatment of TB. Ultimately we hope to draw lessons and experiences from this project and develop policy implications for the continued improvement of TB detection and treatment in China.

\section{Methods}

\section{Study design and setting}

The China NHC-Gates Foundation TB program Phase $\otimes$ was implemented in three provinces: Zhejiang, Jilin, and Ningxia. These provinces represent high, middle and low-levels of economic development. We present the demographic and socioeconomic characteristics of each of the provinces in Appendix 1.

The project implemented a comprehensive package of interventions in December 2016 to improve TB care and control. The following interventions were all implemented: 1) Strengthen TB suspect screening and referral system 2) Expand the use of new TB diagnostic technologies such as rapid molecular tests (RMT) 3) Use rational treatment regimens based on DST results 4) Strengthening public health functions of TB designated hospitals and 5) Improve collaboration between CDC, TB hospitals and primary health clinics 6) An e-learning system and other capacity-building activities on TB diagnosis and clinical treatment to improve the capacity of diagnosis and treatment for TB 7) Financing mechanism to reduce patients' financial burden in seeking care for TB. 
For our evaluation sites, we selected 2 prefectures from each of the three provinces, and then two counties from each prefecture based on their level of economic development and TB epidemic features. In total, we evaluated 12 sites. We followed a before-and-after design to assess the changes in the access to and quality of TB related diagnosis and treatment. We collected 2015 data in July 2016 to use as our baseline and 2018 data in July 2019 for our final evaluation.

\section{Data collection}

We performed routine data collection and conducted medical records review in July 2016 and JulyAugust 2019 in our study sites. Specifically, we used the TB Information Management System (TBIMS), a routine registration data operated by CDCs across China, to obtain TB and DRTB patient information for those registered between January 01-December 31, 2015 and January 01-December 31, 2018. Patients with pulmonary tuberculosis diagnosis were eligible for analysis. From TBIMS, we extracted the following information: demographic information such as gender, age and registered location of the patient, diagnosis, bacteriological testing results including sputum smear, sputum culture, and RMT and drug susceptibility tests (DST) and results. Patients with diagnosis of extrapulmonary tuberculosis or tuberculous pleurisy were excluded from analysis. For the baseline analysis for data from 2015, 2,904 TB patients were eligible and included in the analysis. For the final evaluation in 2018, 2,606 TB patients were eligible and included in the analysis.

We conducted medical records review in 2016 and 2019 in our study sites for our baseline and final evaluation. We used the same checklist developed by TB clinical experts and public health experts to perform the review on medical records. The checklist included information about demographics, symptoms, diagnosis, bacteriological testing results including sputum smear, sputum culture, RMT, Chest X-ray and CT examinations, immunological examinations, differential diagnosis information, treatment regimens and outpatient and inpatient records. Please refer to Appendix 2 for details about the checklist. We reviewed medical records for pulmonary TB patients diagnosed between January 01- December 31, 2015 and January 01- December 31, 2018. Patients with diagnosis of extrapulmonary tuberculosis or tuberculous pleurisy were excluded from the analysis.

According to our inclusion and exclusion criteria, staff from TB designated hospitals extracted the eligible medical records, starting with the records with most recent date, until they reached the required sample size. In each TB designated hospital of study sites, eligible medical records were gathered by the hospital first. Then a medical doctor specialized in TB and a research assistant with training before the survey, reviewed the medical records and completed the checklist for each patient.

In short, we plan to collect 1) outpatient medical records of 100 TB patients, who have completed their treatment for TB, from each county-level TB designated hospital to examine whether the patients have received sputum examinations and chest X-ray or CT examinations, routine blood tests, liver and renal function tests properly; 2 ) inpatient medical records of 55 smear-negative TB patients (or patients without bacteriological confirmation) from each county, to assess the quality of diagnosis for TB patients without 
bacteriological confirmation; and 3) inpatient medical records of 40 TB patients (including smearnegative TB patients) to assess the use of second line TB medicines.

In counties where the number of eligible TB patients is smaller than the required sample size, all eligible medical records were reviewed. Similarly, in prefectures with less than 60 DRTB patients, all medical records were reviewed. In total, we reviewed 829 records in 2015 and 1,185 records of TB patients in 2018. We reviewed 235 and 356 inpatient medical records for TB patients without bacteriological confirmation, respectively in 2015 and 2018; and we reviewed 286 and 690 eligible medical records of TB inpatients from 2015 and 2018, respectively (Please refer to Appendix 3 for more information about sampling).

\section{Measurements and definitions}

We adopted measurements for the access and quality of diagnosis and treatment based on the WHO TB Report and China's TB guidelines[18].

\section{1) Access to quality diagnosis}

Quality of diagnosis was defined based on the 2008 Guidelines for the Implementation of Tuberculosis Control Programmes in China ("Guideline") as patients who receive proper diagnostic test(s), including TB related tests such as the sputum culture and RMT, and other tests such as Chest X-ray or CT examinations, and immunological examinations[19]. We used the TBIMS data to collect information about the different types of TB diagnostic tests that were performed among smear-negative patients and bacteriological confirmed TB cases, and coverage of DST for TB patients. Then we compared the information we collected with Guideline to assess proportion of TB patients received appropriate tests. Proper use of DST and the testing time reflected the quality of the diagnosis. We included the following dimensions for quality of the diagnosis:

1. Diagnosis for smear-negative TB patients: We report proportion of smear-negative patients that have received at least one of culture or RMT. We then report the coverage of smear, culture and RMT among smear-negative patients, respectively (Source: TBMIS).

2. Diagnosis for bacteriologically confirmed TB patients: Bacteriologically confirmed TB case is defined as patients from whom a biological specimen is positive by either smear microscopy, culture or rapid molecular tests (RMT) such as the Xpert MTB/RIF® assay. We report proportion of bacteriologically confirmed TB case. Then we report share of smear microscopy positive, culture positive and RMT positive among bacteriologically confirmed TB cases (Source: TBMIS).

3. Diagnosis for drug susceptibility: Diagnosis for drug susceptibility was measured by drug susceptibility testing (DST) coverage for bacteriologically confirmed TB patients. We report proportion of bacteriologically confirmed TB cases tested for drug resistance by phenotypic DST or RMT. Then we present share of RMT and phenotypic DST among bacteriological confirmed TB cases (Source: TBMIS). 
4. Adequate diagnostic services among TB patients: According to the Guideline, we developed two indicators to assess the proportion of TB patients who have received adequate diagnostic services.

According to the Guideline, TB patients should receive at least one of sputum examinations (sputum smears or sputum culture) and at least one of the Chest X-ray or CT examination. Among all reviewed outpatient medical records of TB patients, we reported the percentage of TB patients who received these recommended tests (Source: medical record review).

According to the Guideline, TB patients without bacteriological confirmation are recommended to receive all of the following tests: a) three sputum smears with negative results; $b$ ) chest $\mathrm{X}$-ray or CT examinations showed lesions consistent with active tuberculosis, and c) one of the following: typical TB symptoms; positive result of immunological tests from tuberculosis skin test (PPD), anti-tuberculosis antibody test or interferon-gamma release assays (IGRAs); differential diagnosis to rule out other diseases including tumor marker test,exfoliated cell examination, bronchoscopy, lung biopsy and general antibiotics treatment. Among all reviewed inpatient medical records of TB patients, we reported the percentage of TB patients who received these recommended tests, breaking down into asymptomatic pulmonary TB patients and symptomatic pulmonary TB patients, respectively. Suspicious TB symptoms include cough, expectoration, hemoptysis and fever. Proportions of different types of diagnostic tests used by TB patients without bacteriological confirmation can be found in Appendix 4 (Source: medical record review).

\section{Rational use of clinical services}

We collected data from medical records on two indicators to determine whether or not a patient received rational clinical services. Specifically, our analysis focused on the irrational use of second line TB medication, and the follow-up examinations during the whole treatment course.

1. Proportion of TB patients treated with irrational second-line drugs (SLDs): among TB patients with inpatient medical records, proportion of TB patients received irrational second line anti-TB drugs during treatment. Irrational SLDs treatment was defined by 1) using SLDs as anti-inflammatory treatment before obtaining drug susceptibility testing results; 2) using SLDs to replace Pyrazinamide without specifications (Source: medical record review).

2. Proportion of TB patients receiving recommended follow-up examinations during treatment course: according to the Guideline, the following examinations (with specific time frames) that mainly to monitor the adverse reactions of TB treatment have been analyzed: blood routine test and routine urine test before treatment and one-month completion of treatment; liver and renal function test before treatment, each month during enhanced treatment, and whenever patient has nausea, vomiting or liver pain[19]. We report proportion of TB patients receiving recommended follow-up examinations during treatment course, proportions of patients receiving recommended routine blood tests, liver and renal function test, and routine urine test, respectively. Specifically, seven follow-up tests during treatment course indicated properly follow-ups while three tests during was the minimal requirements (results presented in Appendix 3. Proportions of different types of tests used by TB patients can be found in Appendix 3 (Source: medical record review). 


\section{Analysis}

We used a before-and-after approach to assess the changes in access to and quality of diagnosis and treatment for TB in all study sites. All indicators were assessed for years 2015 and 2018 i.e., our baseline and end line. We also provided national average of selected indicators as comparison when publicly available. We compared the changes between 2015 and 2018 by using Student's $t$ test on continuous variables and Pearson $\chi^{2}$ tests or Fisher's exact tests on categorical variables. We used two-sided tests with $95 \% \mathrm{Cl}$ for all analysis. Data was double entered by using EpiData software (version 3.1). Statistical analyses were done with SPSS version 22.0.

\section{Quality assurance}

Multiple approaches were taken to ensure quality assurance in both study design and data collection. We hosted three rounds of expert consultation meetings to determine both the plan for data collection and the design of the checklist for the review of medical records. Experts included clinical and public health experts in TB diagnosis and treatment in China, staff from the TB management unit of China CDC and local CDC, and international experts from academic institutions. All of the data collection instruments, tools and procedures were tested before the investigation began. Investigators engaged in the data collection received proper training from clinical and public health experts in TB diagnosis and treatment. All data were cleaned after collection and underwent logical checks for accuracy and consistency.

\section{Ethical considerations}

The study was reviewed and approved by the Duke Campus IRB (\#D0877 and \#2017 - 0768). The China NHC-Gates Foundation TB Phase III project received the ethical approval from China CDC (No.201626). All findings are reported at disaggregated level.

\section{Results}

\section{Access to quality diagnosis}

\section{1) Diagnosis for smear-negative TB patients}

Access to quality diagnosis for smear-negative TB improved substantially over the evaluation period. Coverage of smear culture and RMT among smear-negative patients increased from $34.7 \%$ in 2015 to $87.4 \%$ in 2018. All three provinces have significantly improved diagnosis services during the study period (Fig. 1). The proportion of smear-negative TB patients who received sputum culture and RMT in addition to the smear test has increased from 0-34.3\%. RMT was not widely used in 2015: no patients were diagnosed by RMT in 2015. By 2018, over half (50.4\%) of patients used RMT (Fig. 2).

\section{2) Diagnosis for bacteriologically confirmed TB patients:}

The proportion of bacteriologically confirmed TB case increased substantially during the study period and the RMT has been widely used in 2018. The percentage of pulmonary TB cases confirmed by 
bacteriology increased from 36.2\% in 2015 to $51.7 \%$ in 2018 (Fig. 3) with a growth rate of $15.5 \%(\mathrm{P}<$ 0.05). Compared to the national average, two provinces (Zhejiang and Jilin) performed above the national average (30\%) in 2015. By 2018, all three provinces had a much higher proportion of bacteriologically confirmed TB cases than the national average[6, 20]. Ningxia made the largest progress, with $22.0 \%$ increase, followed by Jilin (16.3\%), and Zhejiang (14.5\%). Zhejiang had the highest percentage of pulmonary TB cases confirmed by bacteriology (42.3\%) amongst the three while in 2018, all three provinces had nearly $50 \%$ or even higher proportion of pulmonary TB cases confirmed by bacteriology.

The use of different diagnostic tests in identifying bacteriologically confirmed TB case has shifted over time (Fig. 4). In 2015, over $92 \%$ bacteriologically confirmed TB cases were diagnosed through sputum smear test and the remaining $7.3 \%$ were confirmed by sputum culture. In 2018 , the RMT was used to diagnose $20.3 \%$ of bacteriologically confirmed TB cases. There was increased share of TB cases confirmed by sputum culture in 2018 while less than $68 \%$ patients were diagnosed by sputum smear test. Ningxia had the highest proportion of bacteriologically confirmed TB cases diagnosed by sputum culture in 2015 (12.9\%). By 2018, this proportioned dropped to only $4.1 \%$. In $2018,22 \%$ of bacteriologically confirmed TB patients in Ningxia were diagnosed by RMT, followed by nearly $20.4 \%$ in Jilin and $19.1 \%$ in Zhejiang.

\section{3) Diagnosis for drug susceptibility:}

DST coverage for bacteriologically confirmed TB patients doubled from $40.2 \%$ in 2015 to $81.8 \%$ in 2018 (Fig. 5). Zhejiang province maintained over $80 \%$ DST coverage during the study period, while both Jilin and Ningxia increased DST coverage substantially, from $9.4-72.3 \%$ and $40.9-87.6 \%$, respectively. Compared to the national average, all three provinces had DST coverage levels that were slightly higher than the national average in 2015. At the end of the study period, all three had much higher DST coverage than the national average[6]. While only $10 \%$ of patients received RMT at the beginning of the study period, over 75\% patients did in 2018 (Fig. 6).

\section{4) Adequate diagnostic services among TB patients}

The proportion of TB patients receiving adequate diagnostic services increased from $85.3-95.4 \%$ during the study period (Fig. 7). All three provinces improved this indicator by at least $8 \%$ between study period and Ningxia had highest proportion of TB patients receiving adequate diagnostic services among the three.

In $2015,75 \%$ of all TB patients without bacteriological confirmation received the recommended diagnostic services outlined in the guidelines. By 2018 , this share was over $86 \%$. Among asymptomatic TB patients (24.3\% in 2015 and 18.3\% in 2018), the proportion of smear-negative TB patients that received the recommended diagnostic services as outlined in the Guideline increased substantially from $18.2 \%$ in 2015 to $63.1 \%$ in 2018 (Fig. 8). In comparison, among TB patients with symptoms $(76.7 \%$ in 2015 and $81.7 \%$ in 2018), the overall proportion of smear-negative TB patients that received the 
recommended diagnostic services by Guideline declined slightly. The proportion of different tests used by TB patients can be found in Appendix 3.

\section{Rational use of TB clinical services}

\section{1) Proportion of TB patients treated with irrational second-line drugs (SLDs)}

The proportion of TB patients treated with irrational SLDs increased from 12.6\% in 2015 to $19.9 \%$ in 2018 (Fig. 9). Over $27.7 \%$ of TB patients in Zhejiang received SLDs in 2018, followed by Jilin and Ningxia. Zhejiang also witnessed the largest increase in proportion of TB patients treated by SLDs between 2015 and 2018 , followed by Jilin.

\section{2) Proportion of TB patients receiving recommended follow-up examinations during treatment course}

The proportion of TB patients receiving the recommended follow-up examinations during the treatment course increased between 2015 and 2018 (Fig. 10). The proportion of patients receiving all recommended tests increased from $21.4 \%$ in 2015 to $64.6 \%$ in 2018 . While all three provinces have made progress, more patients received all of the recommended tests in Zhejiang than the other two provinces in both 2015 and 2018. Across different types of tests, the proportion of patients that received proper routine blood tests saw the largest improvement from $27.7 \%$ in 2015 to $71.9 \%$ in 2018. A higher proportion of patients in Zhejiang received proper routine blood tests and liver function tests than the other two provinces. A higher proportion of patients in Ningxia received routine urine tests than Zhejiang and Jilin. The proportion of patients that received the minimal recommended tests can be found in Appendix 3 . We also present the proportion of different tests used by TB patients in Appendix 4.

\section{Discussion}

Our study found that between 2015 and 2018, access to quality diagnosis for TB improved substantially in the study sites, mainly attributed by the increased use of RMT and improved diagnostic services for smear-negative patients. The coverage of smear culture and RMT among smear-negative patients more than doubled from $35 \%$ in 2015 to $87 \%$ in 2018 . The percentage of pulmonary TB cases confirmed by bacteriology increased from $36 \%$ in 2015 to $52 \%$ in 2018 . RMT was widely used in 2018: over half $(50.4 \%)$ of the smear negative patients used RMT and $20.3 \%$ of bacteriologically confirmed TB cases was diagnosed by RMT. DST coverage for TB patients also doubled between 2015 and 2018 and the proportion of TB patients receiving adequate diagnostic services increased from 88-96\%, respectively. Among all TB patients without bacteriological confirmation, over $86 \%$ received the recommended diagnostic services at the end of the study period, an improvement from $75 \%$ prior to project implementation. Compared to patients with symptoms, asymptomatic TB patients without bacteriological confirmation witnessed greater improvement in receiving recommended diagnostic services (18.2\% in 2015 to $63.1 \%$ in 2018). However, access to rational treatment had mixed findings: the proportion of TB patients treated with irrational SLDs increased between 2015 to 2018 while the 
proportion of patients receiving a recommended follow-up tests during treatment course improved substantially. Regional disparities remained but the gaps were narrowed for almost all indicators.

We observed significant improvements in access to quality TB diagnosis measuring by various indicators after the implementation of the China - Gates Project Phase III. The introduction of RMT is one of the major contributors of improvement in access to quality TB diagnosis. Similar to other studies, our study proved that the introduction of RMT increased the diagnosis of DR-TB[21-23]. The impact of China NHC- Gates Foundation TB Project Phase III was beyond the application of RMT. The capacity training activities have improved the access to quality diagnosis of bacteriologically confirmed TB patients and TB patients without bacteriological confirmation as well. And improved diagnosis is critical for achieving desired treatment results.

China introduced DOTs in the 1990s, which effectively improved the completion rate for TB treatment[24]. However, the detection of TB and DR-TB remains a barrier for furtherer improvement to TB control in China[25]. Compared to the global average of $56 \%$ in 2015, the proportion of TB cases confirmed by bacteriology in China was only 30\%[20]. And the majority of TB cases in China are diagnosed through symptoms, chest radiography, pathology or histology, which have low specificity and could potentially delay treatment for TB[26]. Parsons LM reported that the specificity of sputum smear was largely determined by the quality of sputum sample and experiences of the diagnostic staff while the sputum culture was also limited by capacity, time, and the devices[27]. Without reliable and timely diagnosis, the treatment for TB could potentially be delayed and lead to further spread of TB[28]. Additionally, the lack of precise diagnosis for TB has led to low detection rates of DR-TB patients.

New TB diagnostic devices haven't been widely introduced in China for many reasons. There is no domestic funding to support the implementation of GeneXpert in most regions of China. Additionally, the number of staff trained to perform TB diagnosis is insufficient[29]. The RMT was scaled up through the China NHC - Gates Foundation TB Project Phase III in the three project provinces, which have contributed to the improvement of detecting TB patients. The training activities also increased the capacity of staff involving in the TB diagnosis. The proportion of bacteriologically confirmed TB cases in the three project provinces (36.2\%) was slightly above the national average (30.0\%) in 2015 , while in 2018 , proportion of bacteriologically confirmed TB cases in the three provinces (52\%) has almost reached the global average (54\%). The application of RMT essentially improved the diagnosis of TB, which further promoted the treatment and control of TB. We also observed the regional gaps in access to quality have been narrowed.

Infectious disease control is a public good and should be a top priority for the government. Our findings indicate that improved quality and reduced regional disparity of TB diagnosis by using RMT and it is necessary to develop a sustainable domestic financing mechanism to further scale up the RMT across the country.

Unfortunately, access to rational treatment hasn't made substantial progress. Irrational use of medicine has been a long-lasting issue in China for many reasons[30]. The mark-up from medicines has provided 
adverse financial incentives for providers to prescribe expensive drugs. The situation is even worse for TB. Under current health system in China, TB services are provided at TB-designated hospitals in most regions. However, essential first-line TB drugs, smear tests and chest X-rays are fully subsidized by government and are provided for free. To obtain mark-up from drugs, second-line drugs become more popular. Meanwhile, the wide coverage of health insurance schemes in China also reduce the financial burden of patients who are able to afford more services, including auxiliary medications and follow-up tests.

Irrational treatment could add financial burden to patients. It could lead to health concerns and even drugresistance. Our findings on the increasing use of SLD call for more attention on the regulation of clinical treatment for TB. It also suggests the important role of the health system in implementing interventions. Financing policies, along with other support policies, should be implemented to promote rational treatment for TB.

Our study attempted to evaluate the impacts of a comprehensive TB control models with a wide range of interventions on access to quality diagnosis and rational treatment. We employed a set of indicators to track the changes of access to quality diagnosis and rational treatment, but our observation was limited within the TB care system and the availability of data from TBIMS and medical records. No considerations have been given to other factors or the overall health system. Several study sites have relatively small number of TB patients, which is another limitation of our study. Finally, the data from TBIMS is designed for routine administration. It does not necessarily have robust quality assurance, and errors in data entry cannot be ruled out.

\section{Conclusions}

Access to quality TB diagnosis and follow-up services has been improved substantially in the study sites between 2015 and 2018 primarily due to the implementation of the China NHC- Gates Foundation Tuberculosis Control Project. The introduction of RMT is one of the major contributors of improvement in access to quality TB diagnosis. Access to rational treatment hasn't made substantial progress and irrational use of SLDs remains a problem. Financing policies and other support policies should be implemented to promote rational treatment for TB.

\section{Abbreviations}

TB: Tuberculosis; NHC: The Chinese National Health Commission; China CDC: Chinese Center for Disease Control and Prevention; BMGF: Bill and Melinda Gates Foundation; WHO: World Health Organization; TBIMS: Tuberculosis Information Management System; RMT: Rapid Molecular Tests; SLDs: Second-line Drugs; DALYs: Disability-Adjusted Life Years; DOTS: The Directly Observe Treatment Strategy; DST: Drug Susceptibility Test; MDR-TB: Multidrug-resistant Tuberculosis; CT: Computed tomography; ADR: Adverse Drug Reactions; EMMs: Electronic Medicine Management System 


\section{Declarations}

Acknowledgements

The authors of the paper would like to thank all the staff from designated TB hospitals and CDCs in study sites for their assistance in the data collection.

\section{Authors' contributions}

TS, MG and QZ conceptualized the paper. ZL, WM and QZ analysed and interpreted the data and draft the manuscript. All authors contributed to the drafting of the manuscript. All authors read and approved the final manuscript.

\section{Funding}

The whole study was supported by the Bill and Melinda Gates Foundation. The funders had no role in study design, data collection and analysis, decision to publish, or preparation of the manuscript.

\section{Availability of data and materials}

The datasets generated and analysed during the current study are not publicly available due to the regulations of China CDC. Readers of the article need to discuss with China CDC and obtain their permission before the release of the dataset.

\section{Ethics approval and consent to participate}

Ethical approval of the study was issued by the Institutional Review Board of Chinese Center for Disease Control and Prevention. Consent form was obtained before individual data collection and could be provided upon request.

\section{Consent for publication}

Not applicable.

\section{Competing interests}

All authors declare that they have no competing interests.

\section{References}

1. Uplekar M, Weil D, Lonnroth K, Jaramillo E, Lienhardt C, Dias HM, Falzon D, Floyd K, Gargioni G, Getahun H et al: WHO's new end TB strategy. LANCET 2015, 385(9979):1799-1801.

2. Department of Economic and Social Affairs: Transforming our world: the $\mathbf{2 0 3 0}$ Agenda for Sustainable Development. Available from: https://sdgs.un.org/2030agenda. Accessd on November 25, 2020. 
3. Wang L, Zhang H, Ruan Y, Chin DP, Xia Y, Cheng S, Chen M, Zhao Y, Jiang S, Du X et al: Tuberculosis prevalence in China, 1990-2010; a longitudinal analysis of national survey data. LANCET 2014, 383(9934):2057-2064.

4. Wang L, Li R, Xu C, Zhang H, Ruan Y, Chen M, Wang D, Dirlikov E, Du X, Zhao J et al: The Global Fund in China: Multidrug-resistant tuberculosis nationwide programmatic scale-up and challenges to transition to full country ownership. PLOS ONE 2017, 12(6):e177536.

5. Wang L, Liu J, Chin DP: Progress in tuberculosis control and the evolving public-health system in China. LANCET 2007, 369(9562):691-696.

6. World Health Organization. Global tuberculosis report 2019. 2019.

7. Hargreaves NJ, Kadzakumanja O, Whitty CJ, Salaniponi FM, Harries AD, Squire SB: 'Smear-negative' pulmonary tuberculosis in a DOTS programme: poor outcomes in an area of high HIV seroprevalence. Int J Tuberc Lung Dis 2001, 5(9):847-854.

8. Lombardi G, Di Gregori V, Girometti N, Tadolini M, Bisognin F, Dal Monte P: Diagnosis of smearnegative tuberculosis is greatly improved by Xpert MTB/RIF. PLOS ONE 2017, 12(4):e176186.

9. Steingart KR, Schiller I, Horne DJ, Pai M, Boehme CC, Dendukuri N: Xpert@ MTB/RIF assay for pulmonary tuberculosis and rifampicin resistance in adults. Cochrane Database Syst Rev 2014, 2014(1):Cd009593.

10. Mao W, Jiang W, Hamilton C, Zhang H, Huang F, Lucas H, Huan S, Tang S: Over- and under-treatment of TB patients in Eastern China: an analysis based on health insurance claims data. TROP MED INT HEALTH 2019, 24(9):1078-1087.

11. Zhao P, Li XJ, Zhang SF, Wang XS, Liu CY: Social behaviour risk factors for drug resistant tuberculosis in mainland China: a meta-analysis. J INT MED RES 2012, 40(2):436-445.

12. Zhao Y, Xu S, Wang L, Chin DP, Wang S, Jiang G, Xia H, Zhou Y, Li Q, Ou X et al: National survey of drug-resistant tuberculosis in China. N Engl J Med 2012, 366(23):2161-2170.

13. Xu L, Chen J, Innes AL, Li L, Chiang CY: Prescription practice of anti-tuberculosis drugs in Yunnan, China: A clinical audit. PLOS ONE 2017, 12(10):e187076.

14. Walzl G, McNerney R, du Plessis N, Bates M, McHugh TD, Chegou NN, Zumla A: Tuberculosis: advances and challenges in development of new diagnostics and biomarkers. LANCET INFECT DIS 2018, 18(7):e199-e210.

15. Opota O, Mazza-Stalder J, Greub G, Jaton K: The rapid molecular test Xpert MTB/RIF ultra: towards improved tuberculosis diagnosis and rifampicin resistance detection. CLIN MICROBIOL INFEC 2019, 25(11):1370-1376.

16. Li R, Ruan Y, Sun Q, Wang X, Chen M, Zhang H, Zhao Y, Zhao J, Chen C, Xu C et al: Effect of a comprehensive programme to provide universal access to care for sputum-smear-positive multidrugresistant tuberculosis in China: a before-and-after study. LANCET GLOB HEALTH2015, 3(4):e217e228.

17. Long Q, Qu Y, Lucas H: Drug-resistant tuberculosis control in China: progress and challenges. INFECT DIS POVERTY 2016, 5:9. 
18. World Health Organization. Global tuberculosis report 2020. 2020.

19. Chinese Center for Disease Control and Prevention. China TB control and prevention plan implementation guide(2018 Edition) 2008. Available from: http://tb.chinacdc.cn/zcfg/sszn/201207/t20120711_64166.htm. Accessd on November 25, 2020.

20. World Health Organization. Global tuberculosis report 2016. 2016.

21. Kabasakalyan E, Davtyan K, Cholakyans V, Mirzoyan A, Kentenyants K, Petrosyan D, Hayrapetyan A, Gupte HA: Change in TB diagnostic profile after introduction of GeneXpert MTB/RIF assay in National TB Program of Armenia, 2013-2017. J Infect Dev Ctries 2019, 13(5.1):22s-27s.

22. Oxlade $O$, Falzon D, Menzies D: The impact and cost-effectiveness of strategies to detect drugresistant tuberculosis. EUR RESPIR J 2012, 39(3):626-634.

23. de Vries G, Tsolova S, Anderson LF, Gebhard AC, Heldal E, Hollo V, Cejudo LS, Schmid D, Schreuder B, Varleva $T$ et al: Health system factors influencing management of multidrug-resistant tuberculosis in four European Union countries - learning from country experiences. BMC PUBLIC HEALTH 2017, 17(1):334.

24. China Tuberculosis Control Collaboration: Results of directly observed short-course chemotherapy in 112,842 Chinese patients with smear-positive tuberculosis. China Tuberculosis Control Collaboration. LANCET 1996, 347(8998):358-362.

25. Sylvia S, Xue H, Zhou C, Shi Y, Yi H, Zhou H, Rozelle S, Pai M, Das J: Tuberculosis detection and the challenges of integrated care in rural China: A cross-sectional standardized patient study. PLOS MED 2017, 14(10):e1002405.

26. Siddiqi K, Lambert ML, Walley J: Clinical diagnosis of smear-negative pulmonary tuberculosis in lowincome countries: the current evidence. LANCET INFECT DIS 2003, 3(5):288-296.

27. Parsons LM, Somoskovi A, Gutierrez C, Lee E, Paramasivan CN, Abimiku A, Spector S, Roscigno G, Nkengasong J: Laboratory Diagnosis of Tuberculosis in Resource-Poor Countries: Challenges and Opportunities. CLIN MICROBIOL REV 2011, 24(2):314-350.

28. Boehme CC, Nabeta P, Hillemann D, Nicol MP, Shenai S, Krapp F, Allen J, Tahirli R, Blakemore R, Rustomjee $\mathrm{R}$ et al: Rapid molecular detection of tuberculosis and rifampin resistance. $N \mathrm{Eng} / \mathrm{J}$ Med 2010, 363(11):1005-1015.

29. Wang Z, Jiang W, Liu Y, Zhang L, Zhu A, Tang S, Liu X: Transforming tuberculosis (TB) service delivery model in China: issues and challenges for health workforce. HUM RESOUR HEALTH 2019, 17(1):83.

30. Mao W, Vu H, Xie Z, Chen W, Tang S: Systematic review on irrational use of medicines in China and Vietnam. PLOS ONE 2015, 10(3):e117710.

\section{Figures}




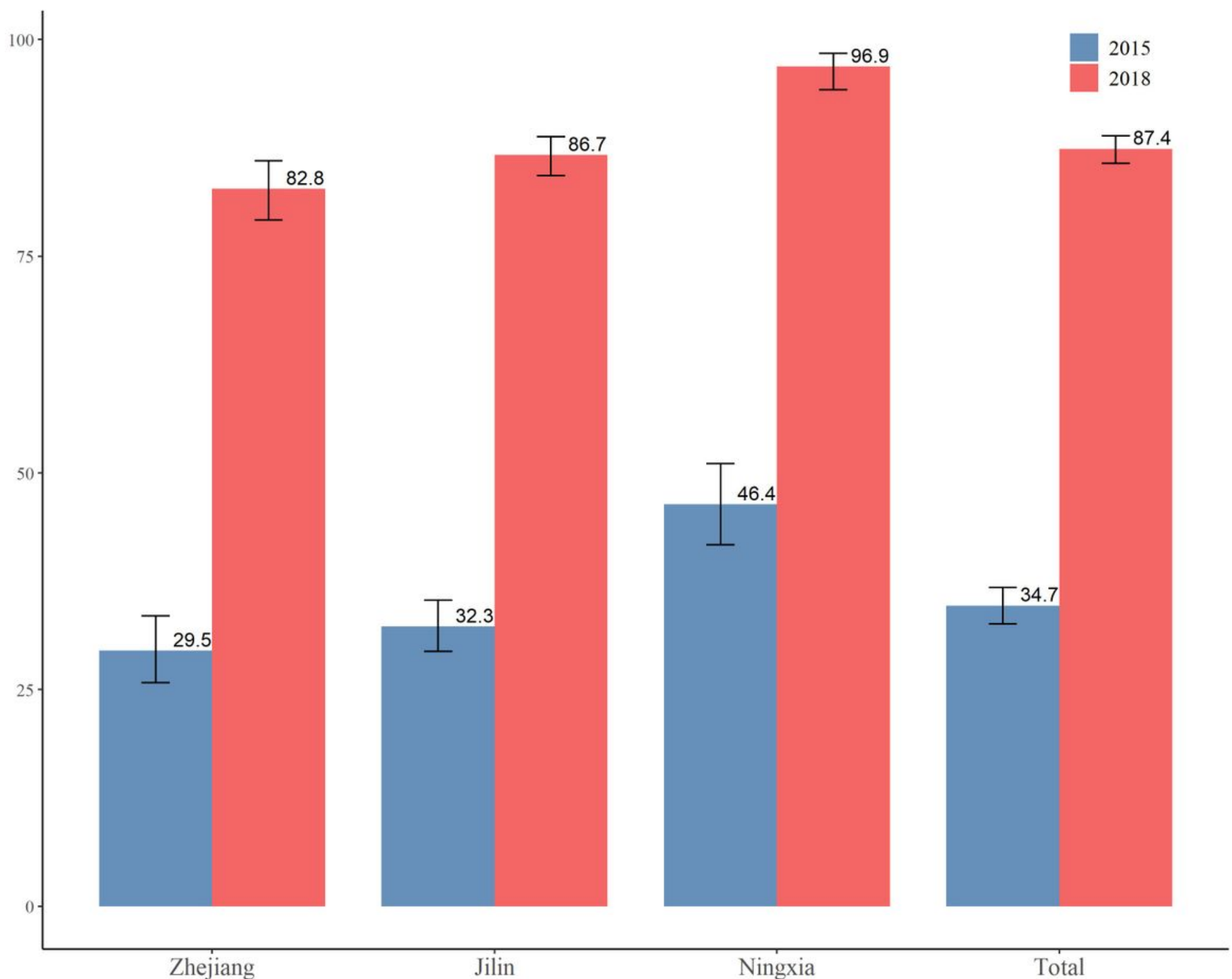

\section{Figure 1}

Coverage of smear culture and RMT among smear-negative patients in 2015 and 2018, disaggregated by province (Source: TBIMS) 


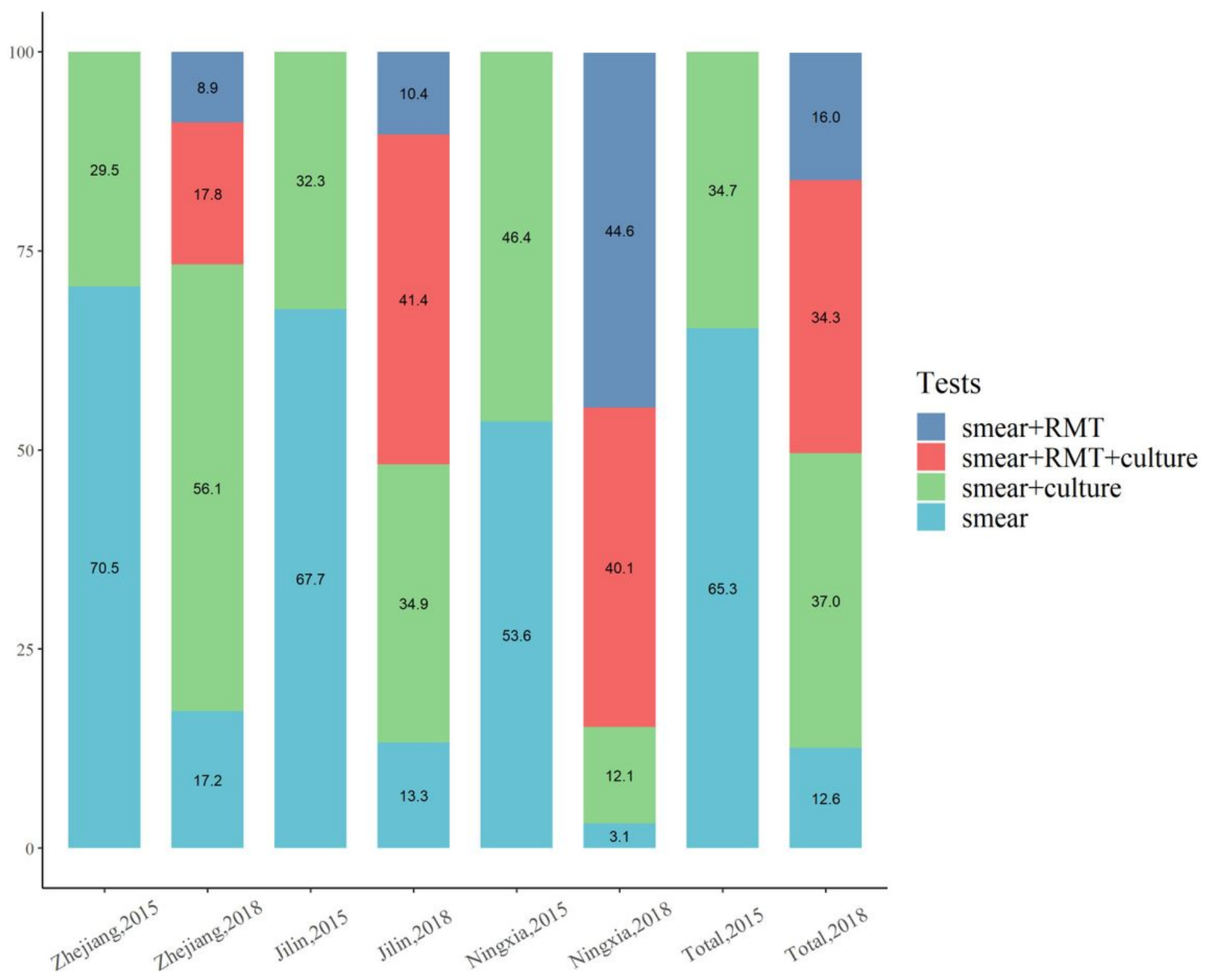

Figure 2

Coverage of different types of TB diagnostic tests among smear-negative patients in 2015 and 2018, disaggregated by province (Source: TBIMS) 


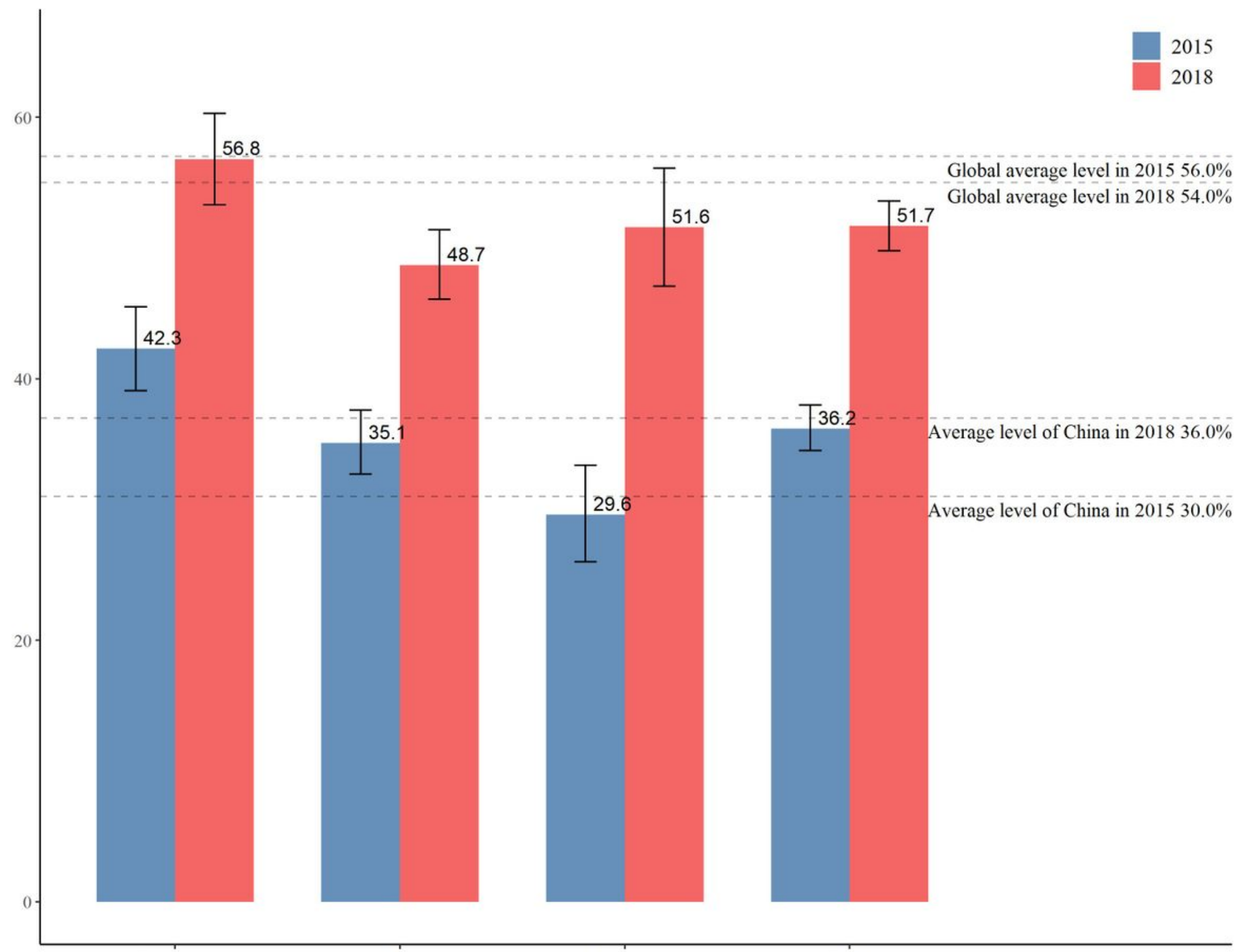

Zhejiang

Jilin

Ningxia

Total

Figure 3

Percentage of pulmonary TB cases confirmed by bacteriology in 2015 and 2018, disaggregated by province (Source: TBIMS) 


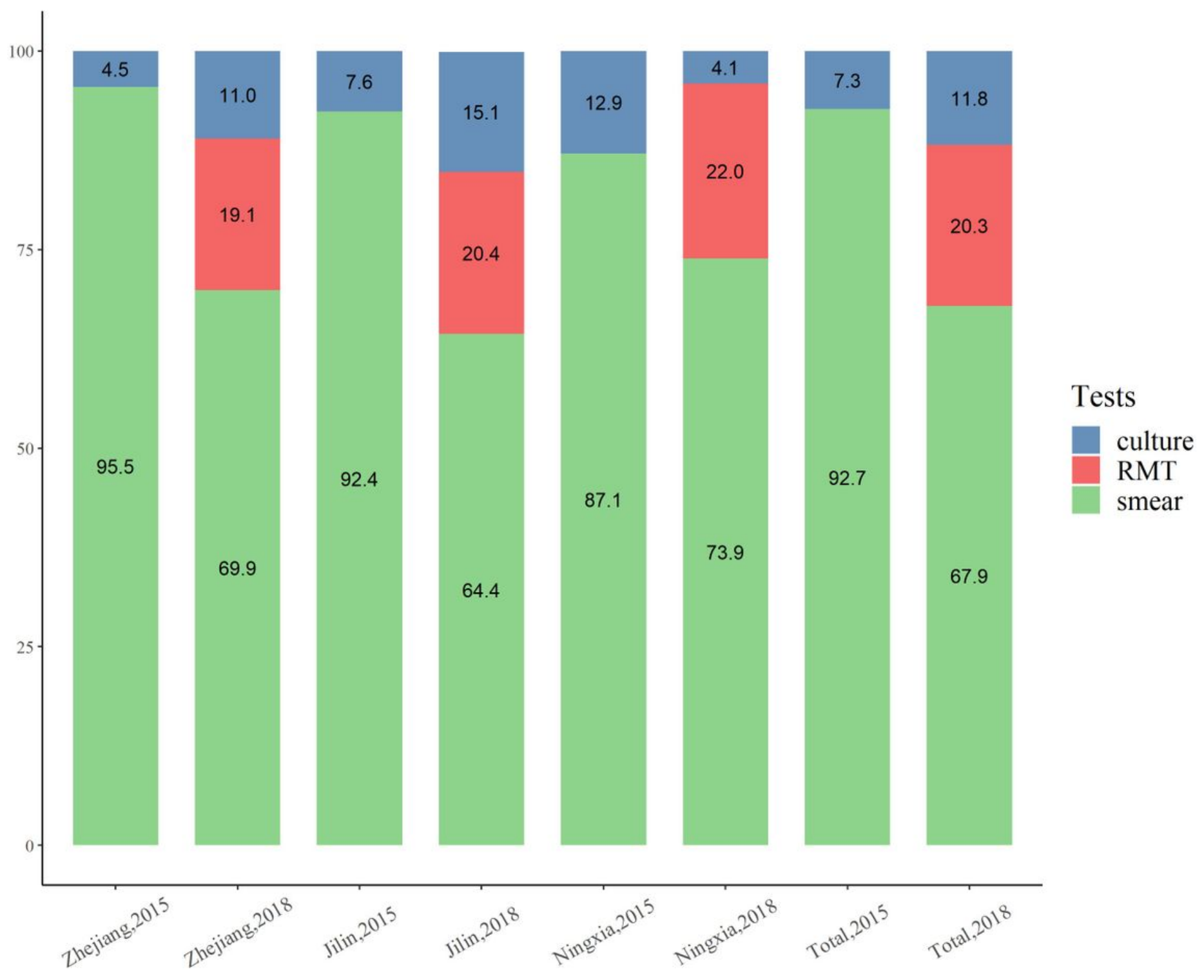

Figure 4

Proportion of diagnostic tests in bacteriological-confirmed TB patients in 2015 and 2018, disaggregated by province (Source: TBIMS) 


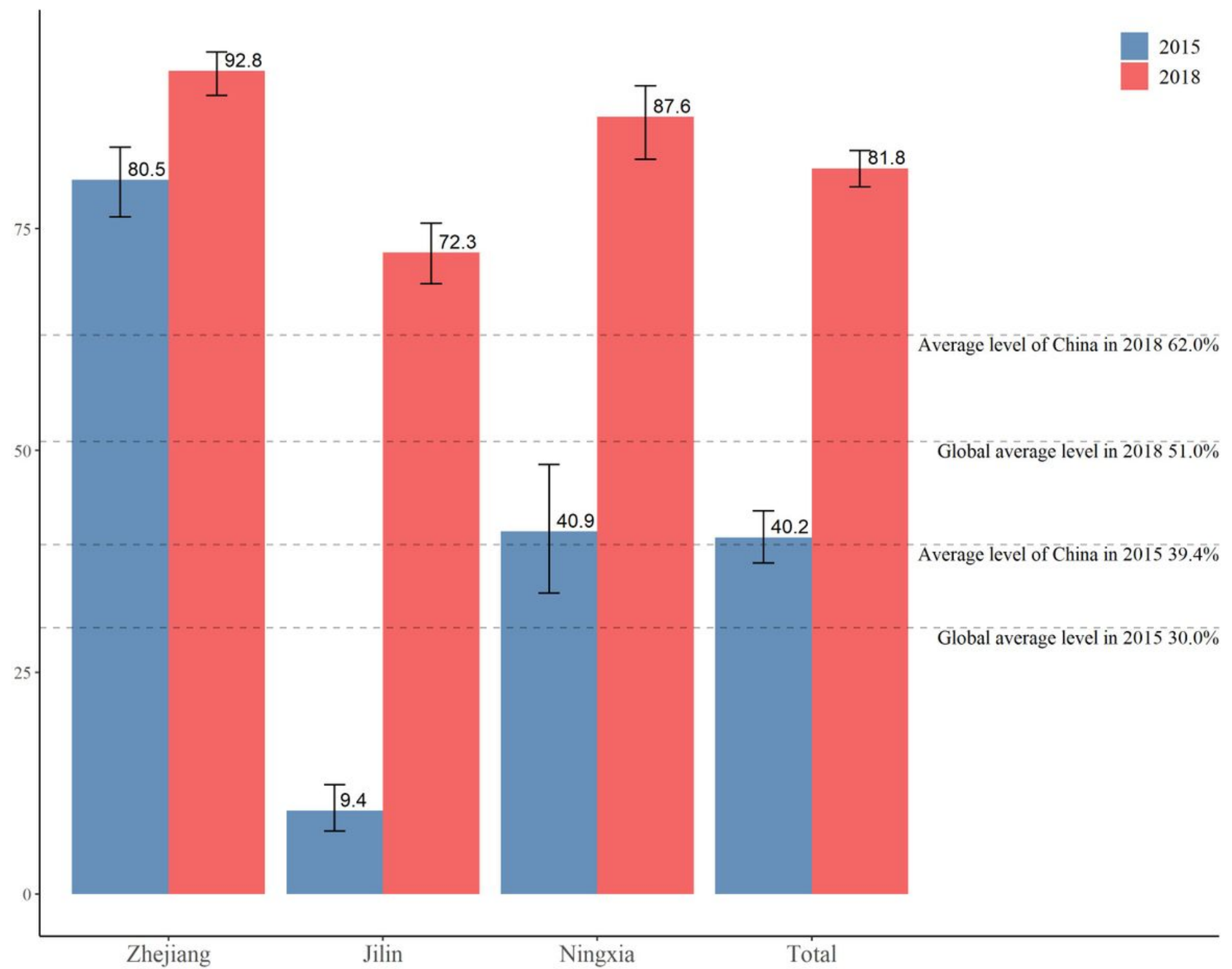

Figure 5

DST coverage for bacteriologically confirmed TB patients in 2015 and 2018, disaggregated by province (Source: TBIMS) 


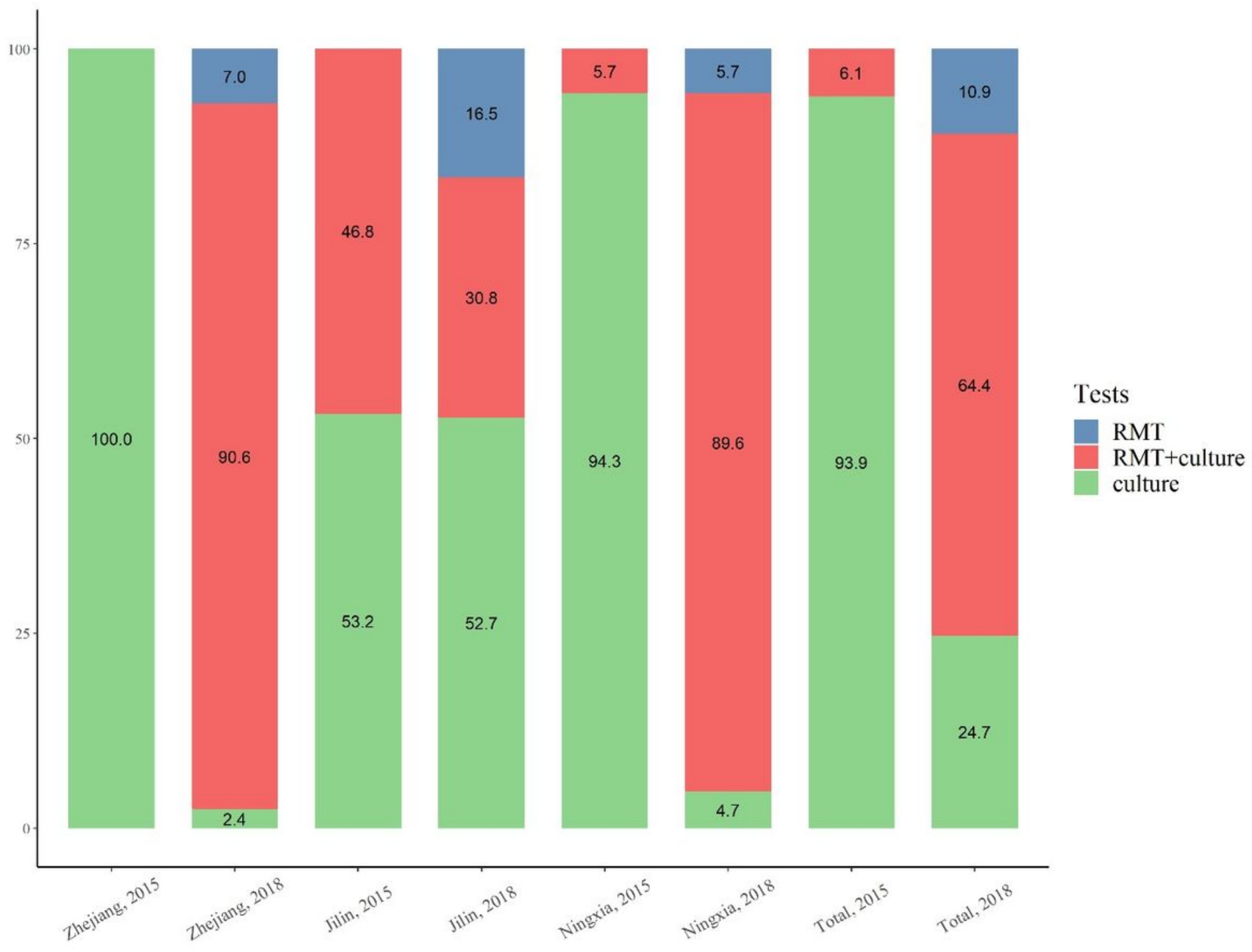

Figure 6

Proportion of diagnostic tests for bacteriologically confirmed TB patients testing for drug susceptibility 2015 and 2018, disaggregated by province (Source: TBIMS) 


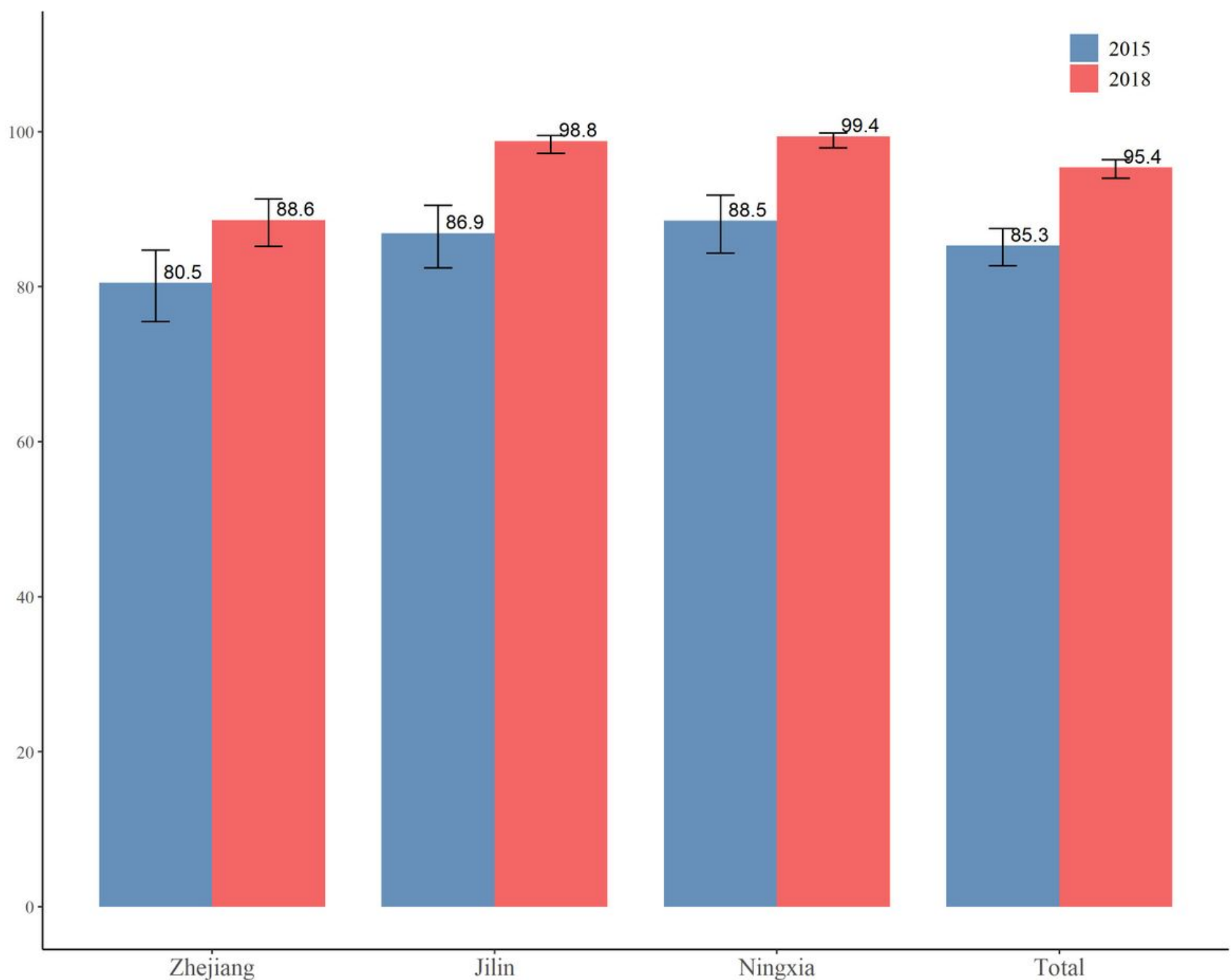

\section{Figure 7}

Proportion of TB patients received adequate diagnostic services in 2015 and 2018, disaggregated by province (Source: medical record review) 

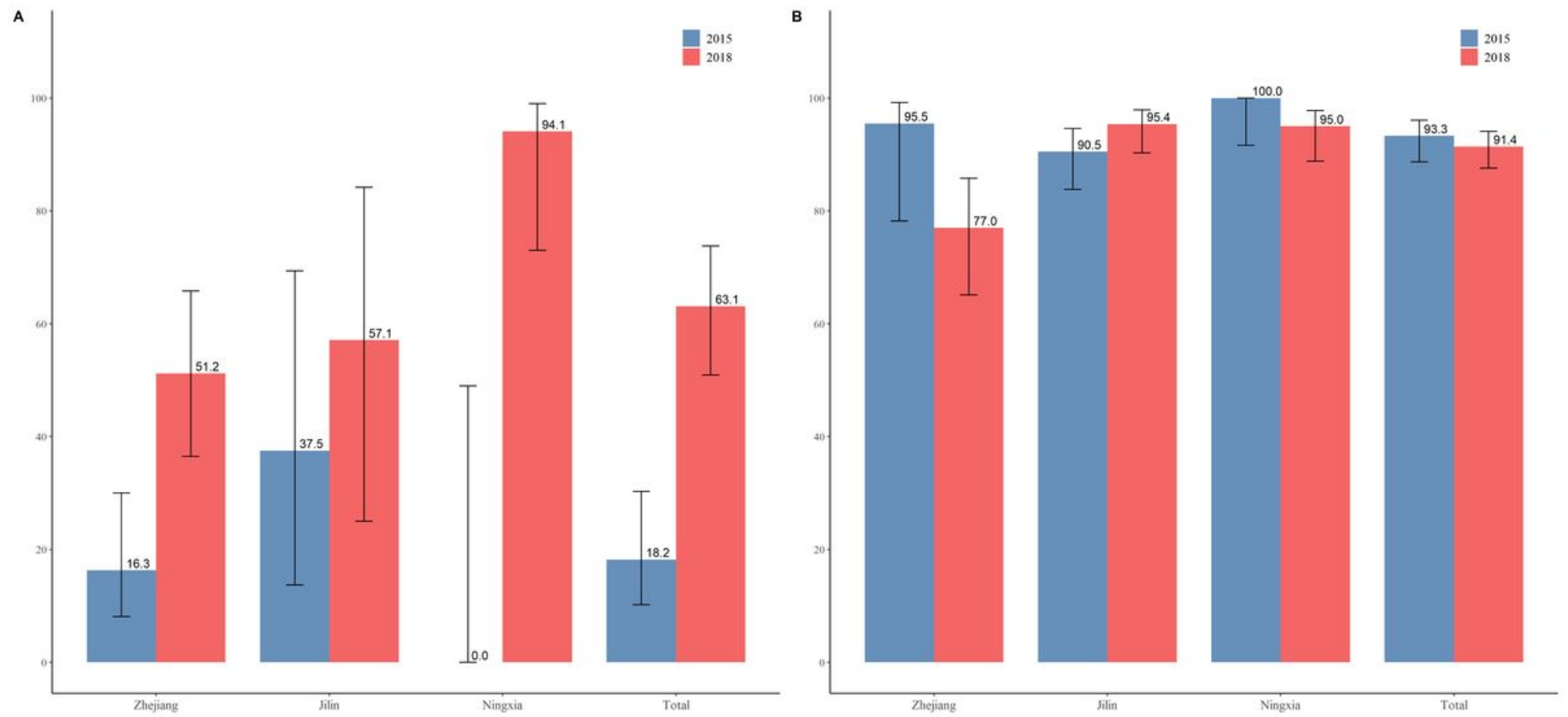

\section{Figure 8}

Proportion of smear-negative TB patients received recommended diagnostic services by Guideline in 2015 and 2018, disaggregated by province A) among asymptomatic pulmonary TB patients B) among symptomatic pulmonary TB patients (Source: medical record review) 


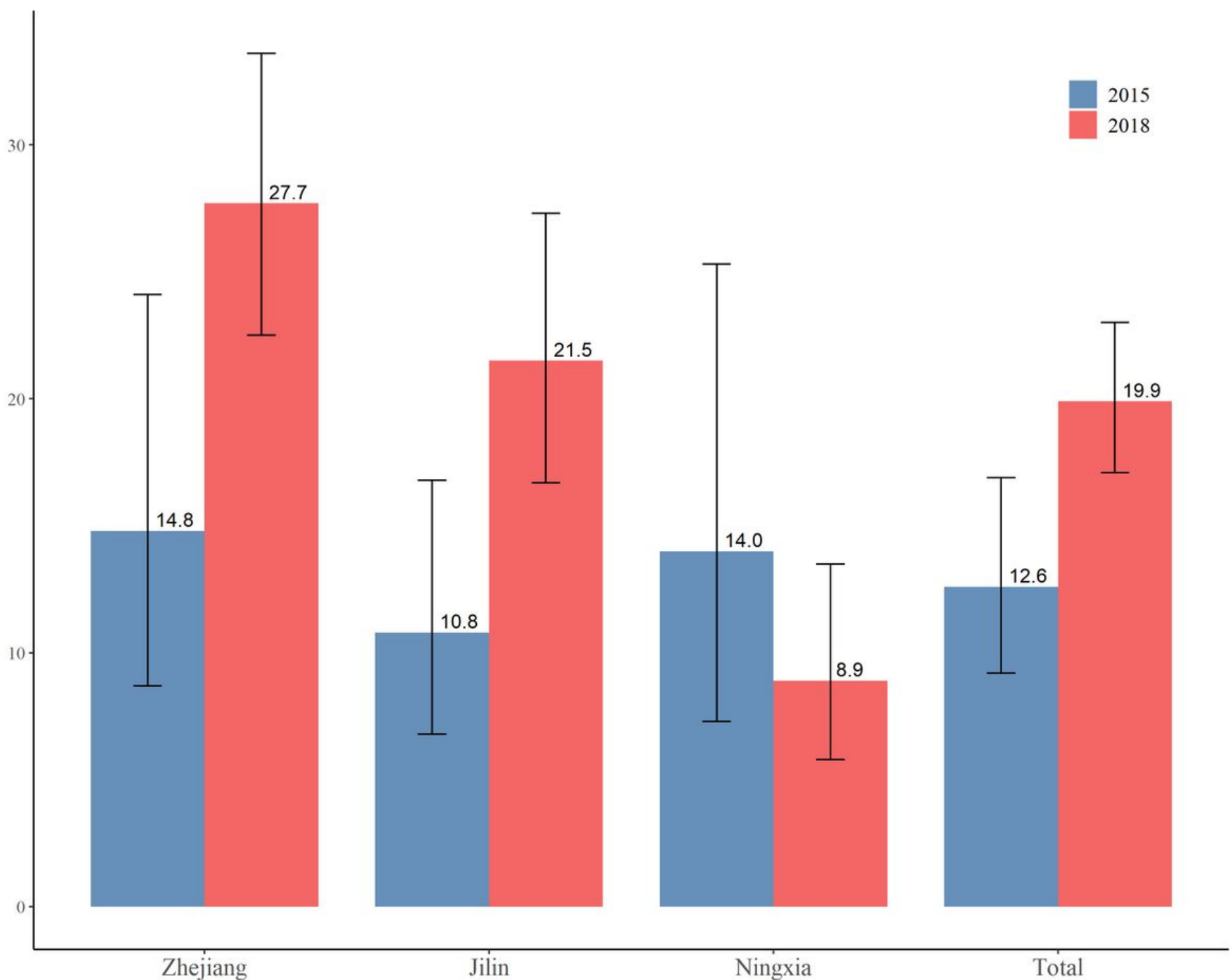

Figure 9

Proportion of drug susceptible TB patients treated with irrational second-line drug in 2015 and 2018, disaggregated by province (Source: medical record review) 

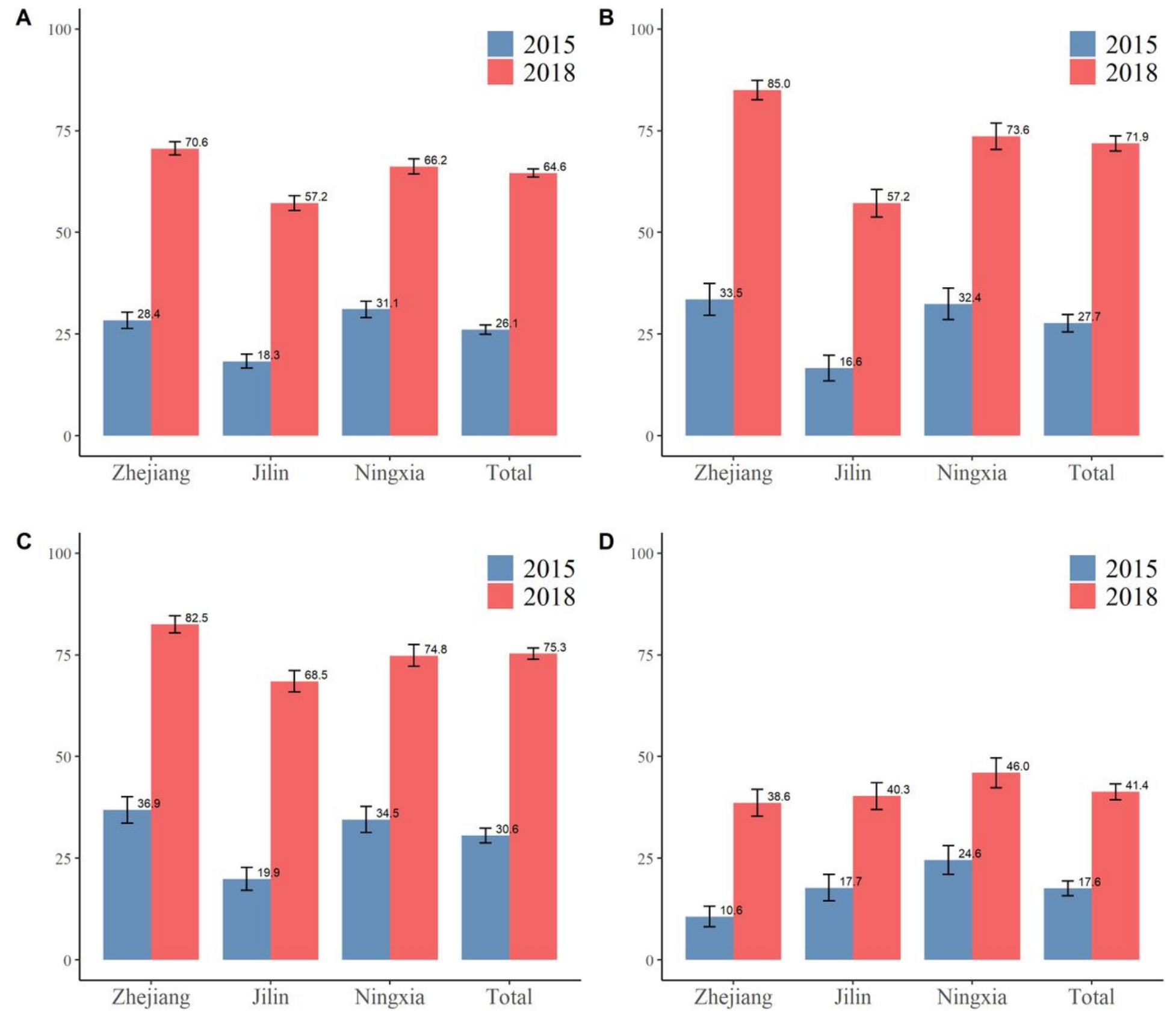

\section{Figure 10}

Proportion of TB patients receiving recommended follow-up examinations during treatment course in 2015 and 2018, disaggregated by province A) all test B) routine blood test C) liver function test D) routine urine test (Source: medical record review)

\section{Supplementary Files}

This is a list of supplementary files associated with this preprint. Click to download.

- Appendix.docx 